\title{
Biologic Agents for Tissue and Bone Regeneration
}

\author{
Hom-Lay Wang*
}

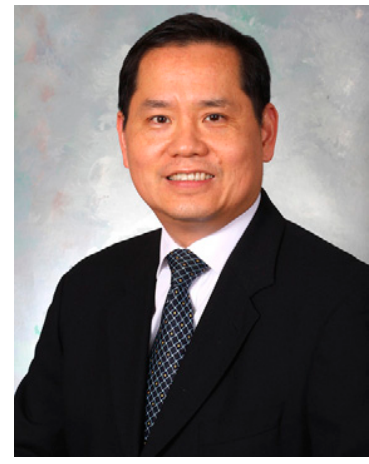

Hom-Lay Wang Guest Editor

Tissue regeneration and bone augmentation have slowly become mainstreams of periodontists' daily practice. Biologic agents which attempt to promote soft and hard tissue repair as well as jump-start the healing process, especially in medically compromised individuals, are an integral part of clinical periodontics. Tissue engineering using biologic agents, such as enamel matrix derivative, platelet-derived growth factor, platelet-rich plasma or platelet-rich fibrin gel, bone morphogenetic protein, fibroblast growth factor, low dosage parathyroid hormone-related protein, or combination therapy, has recently become a focus of research and constant debate among clinicians, especially periodontists. These biologic agents have been tested and show promising results in promoting soft and hard tissue regeneration.

The purpose of this special issue of Clinical Advances in Periodontics is to target this emerging field by inviting individuals who have expertise in this area to share their insights with our readers. All articles contain the most updated information and show how each biologic agent should be used in daily practice. Clinical examples of how each agent can be used in promoting tissue regeneration or bone augmentation are illustrated. In deciding which agent to use, it is important for clinicians to understand the mechanism of each biological modifier and the evidence that exists, as well as other factors such as cost/ benefit ratio, risk/benefit ratio, and long-term stability. Readers are encouraged to understand how these agents work, when to use them, and where they should be used, either alone or in combination, so they can make informed decisions in specific clinical situations.

This special issue begins with an introduction with several focused questions such as: "What biologic agents are available to help promote periodontal regeneration and bone augmentation?"; "How should clinicians decide which biologic agent(s) to use?"; and "What are the approved uses for each agent?". The agents are then linked to their future perspectives in the second paper. In the following papers, the current available agents are introduced with clinical case illustrations as well as current evidence. The final paper demonstrates a combination approach using these agents.

By reading this special issue of Clinical Advances in Periodontics, I hope readers gain a clear understanding of why, how, where, and when these biologic agents should be used in clinical practice to promote tissue and bone regeneration. 